\title{
Desarrollo Local en la Era Post Covid-19
}

\section{Local Development in the COVID-19 era}

\begin{abstract}
Francisco R. Sandoval-Vázquez ${ }^{a}$, José Marcos Bustos Aguayo ${ }^{b}$, Cruz García-Lirios ${ }^{c}$
Abstract:

The objective of this work is to specify a model for the study of the effects of climate change on public health and Local Development. For this purpose, a documentary study was carried out with a sample of sources indexed with ISSN and DOI records published during the period 2010 to 2021. Subsequently, the information was processed in content analysis matrices to establish the axes of discussion and debate topics on the research agenda. Finally, considering the review of the theoretical, conceptual and empirical literature, the model was specified in which the variables of perception, belief, attitude, intention and behaviour were included. In relation to the state of knowledge, the inclusion of emotional and evaluative variables is recommended that allow the contrast of hypotheses related to the effects of floods, droughts, fires and shortage of resources on environmental public health.
\end{abstract}

Keywords:

Climate Change, COVID-19, Local Development, Public Health, Sustainability

Resumen:

El objetivo del presente trabajo es especificar un modelo para el estudio de los efectos del cambio climático sobre la salud pública y el Desarrollo Local. Para tal propósito, se llevó a cabo un estudio documental con una muestra de fuentes indexadas con registro ISSN y DOI publicadas durante el periodo 2010 a 2021. Posteriormente, la información fue procesada en matrices de análisis de contenido a fin de poder establecer los ejes de discusión y temas de debate en la agenda investigativa. Por último, considerando la revisión de la literatura teórica, conceptual y empírica, se especificó el modelo en el que se incluyó a las variables de percepción, creencia, actitud, intención y comportamiento. En relación con el estado del conocimiento se recomienda la inclusión de variables emotivas y valorativas que permitan el contraste de hipótesis relativas a los efectos de inundaciones, sequías, incendios y desabastecimiento de recursos sobre la salud pública ambiental.

Palabras Clave:

Cambio Climático, COVID-19, Desarrollo Local, Salud Pública, Sustentabilidad

\section{Introducción}

El trinomio cambio climático, salud pública y Desarrollo Local suponen una gestión de conocimientos e innovaciones que determinan las relaciones asimétricas entre gobernantes y gobernados $\{1\}$. En este sentido, el objetivo del presente trabajo es delimitar un modelo para el estudio del Desarrollo Local con énfasis en la influencia del cambio climático: desastres naturales, catástrofes ambientales, crisis ecológicas materializadas en inundaciones y sequías con respecto a la salud comunitaria $[2]$. El cambio climático, entendido como un proceso irreversible e ineludible de transformación paulatina o acelerada de la temperatura promedio global afecta directamente la dinámica de comunidades: disminuyendo la calidad de vida y bienestar subjetivo, evidenciando la acción gubernamental corrupta y la hipermetropía ciudadana.

La seguridad pública, a diferencia de la ciudadana o privada, es gestionada por el Estado que, a través de sus instituciones garantiza la estabilidad económica, política y social, pero que al corromperse exacerba los efectos de las crisis ambientales en la vida comunitaria $\{3\}$. Empero, ante inundaciones o sequías, la corrupción del Estado genera indefensión en la ciudadanía y justifica su inacción como

a Universidad Autónoma del Estado de Morelos, https://orcid.org/0000-0002-6086-7197 fsandoval@uaem.mx 
resultado de la difusión de la negligencia de funcionarios que inhibieron la protección civil $[4]$. Se trata de una hipermetropía que se gesta en los medios de comunicación difusores de eventos de riesgo ambiental, financiero o tecnológico asociados a funcionarios o políticos que durante su gestión son señalados como los responsables directos, pero que al ocurrir en un lapso relativamente lejano es reducido a su mínima expresión por la opinión civil.

Es también el caso de los eventos que difunden en tiempo real la televisión e Internet $[5]$. Más allá de concientizar a la ciudadanía, generan una desesperanza y una propensión a vivir un presente hedonista sin importar la disponibilidad de los recursos para las necesidades de las generaciones futuras $\{6]$. Si la salud pública es resultado de un nivel de prevención de enfermedades y atención a las patologías, entonces la hipermetropía es un indicador preponderante, ya que si las inundaciones que ocurren en Asía Pacífico generan paludismo, entonces las audiencias consideran que por la lejanía de las tormentas éstas no pueden afectar su clima o entorno.

Por consiguiente, los efectos del cambio climático sobre la salud pública no sólo inhiben el comercio o el turismo, sino además propician una hipermetropía social que inhibe 1 prevención y desalienta la cooperación o solidaridad ante inundaciones o sequías $[7\rfloor$. Se trata de un proceso en el que el cambio climático, la salud pública y el Desarrollo Local convergen en la hipermetropía como indicador de afectación en las comunidades vulnerables $\{8\}$. Sin embargo, los marcos teóricos y conceptuales que tratan de explicar el proceso o trinomio asumen que la sociedad civil se organiza en esferas para abatir la corrupción o conservar los recursos de un modo indiferenciado y poco integral.

Se trata de grupos ecologistas que emprenden sistemas de preservación ambiental o activistas que buscan evidenciar la corrupción política sin asumir que ambos fenómenos están vinculados [9]. De este modo, la teoría de los nuevos movimientos sociales considera que las problemáticas ambientales son emergentes y consustanciales al cambio climático y en consecuencia, desarrollan estrategias de identidad y participación que los llevan a intervenir directamente en las comunidades y los recursos naturales descartando la acción gubernamental como factor de desabastecimiento de servicios hídricos o energéticos $\lceil 10\rceil$. Por su parte, la teoría de la movilización de recursos asume que el problema estriba en un desbalance entre la disponibilidad hídrica o energética con respecto a las necesidades de las comunidades, por consiguiente, proponen una acción colectiva a partir de un cálculo de demandas y recursos según la cual esperan que la conciencia social se disemine con la sola presencia de la movilización social o la manifestación pública.

La teoría de la privación relativa se aproxima más al fenómeno de la hipermetropía en tanto que plantea un escenario de escasez de recursos y desabastecimiento de servicios públicos que obligaría a la ciudadanía a compararse a sí misma en otros contextos o regímenes, o bien frente a otras sociedades azotadas por las sequías e inundaciones $\{11]$. Empero, la privación relativa no advierte sobre la emergencia del emprendimiento social que es consustancial a la hipermetropía y la indefensión civil [12]. En este sentido, la teoría de la elección racional y la teoría del capital humano explican el proceso racional, deliberado, planificado y sistemático en torno a la toma de decisiones para la prevención o el afrontamiento de las inundaciones o sequías.

Sin embargo, los marcos teóricos y conceptuales revisados asumen que la ciudadanía está en constante cambio y movimiento en función de los fenómenos naturales que indican el cambio climático $\lceil 13\rfloor$. Es decir, las sequías e inundaciones propician una adaptación, asimilación y selección civil a fin de poder reducir los efectos en la calidad de vida y el bienestar subjetivo, pero soslayan las iniciativas y propuestas que se organizan al interior de la sociedad civil [14]. De este modo, la teoría del emprendimiento social comparte el planteamiento de adaptación, asimilación y selección propuesto en los demás marcos teóricos y conceptuales, pero a diferencia de ellos sostiene que la ciudadanía, organizada en esferas civiles, es capaz de construir una responsabilidad social que no sólo prevenga las catástrofes, sino además anticipe escenarios de corrupción política.

Precisamente, el presente trabajo propone un modelo para el estudio del comportamiento sustentable como indicador de Desarrollo Local Sustentable. Para tal propósito, se llevó a cabo un estudio documental con una muestra de fuentes indexadas y con registro ISSN y DOI que fueron publicadas en el periodo que va de 2010 a 2015. Posteriormente, se procesó la información en matrices de análisis de contenido para establecer los indicadores de sustentabilidad local y poder elaborar el modelo de variables observables.

\section{Problemáticas del cambio climático sobre la salud pública y el desarrollo local}

Los efectos del cambio climático sobre la salud pública han sido documentados desde la perspectiva biomédica [15]. La unidad de análisis estriba en las alergias y trastornos nutricionales, así como la afectación a las vías respiratorias $[16]$. En este modelo los medios de comunicación son factores periféricos a la salud pública porque son asumidos como instrumentos de promoción de la salud o prevención de desastres en cuanto protección civil se refiere.

Sin embargo, la emergencia de movimientos civiles en torno al derecho a la salud y los recursos naturales cuestionó el modelo biomédico a la vez que enalteció una propuesta sociológica y política $\left\{18 \int\right.$. En este modelo sociopolítico, el cambio climático es abordado desde las políticas públicas, los programas de atención y las estrategias de intervención local [19]. En materia de salud pública, una política del cambio climático puntualiza la rectoría del Estado como gestor de seguridad alimenticia y sanitaria $[20\rfloor$. Por consiguiente, los instrumentos de gestión son propaganda a 
favor de reformas estructurales que generen inversión y empleo con responsabilidad social.

Sin embargo, la rectoría del Estado ha sido cuestionada debido a la corrupción que los medios de comunicación atribuyen a funcionarios y servidores públicos en cuanto a conflicto de intereses: concesiones discrecionales, favoritismo, opacidad o negligencia [21]. Por otra parte, en el afán de ganar credibilidad, las esferas políticas han implementado ajustes a su estructura burocrática dotándolas de transparencia y rendición de cuentas a fin de involucrar a la ciudadanía no sólo en la observación de la gestión, sino en la emisión de propuestas y establecimiento de acuerdos $[22\rfloor$. De este modo, los efectos del cambio climático sobre el Desarrollo Local suponen cuatro fases e instancias que determinan 16 indicadores.

Por ejemplo, las inundaciones y sequías estarían relacionadas con las capacidades de autogestión e innovación social 'que se generan a partir del incremento de la temperatura promedio de las localidades, la intensificación de tornados, aumento del nivel de los océanos, la cobertura de los medios y la migración de las especies animales y la extinción de las especies vegetales $\{23\rfloor$. A medida que las problemáticas ambientales y os cambios ecosistémicos se exacerban, propician un aumento en el sentido de comunidad: pertenencia y arraigo son factores de preservación del entorno local, pero también de cambios climáticos paulatinos e intensos.

\section{Teorías del desarrollo local}

Los efectos del cambio climático sobre la salud pública y el Desarrollo local han sido explicados desde teorías económicas, políticas, sociológicas y psicológicas, aunque tienen un origen disciplinar, los marcos teóricos y conceptuales son más bien interdisciplinares [24]. Es el caso de la teoría de la centralidad y la periferia que explicita la relación entre economías desarrolladas y países emergentes. La aproximación sostiene que, en el proceso de colonización, el descubrimiento de América redefinió la centralidad de Asía con respecto a la periferia de Europa $\lceil 25]$. El comercio no sólo incentivó las asimetrías entre las regiones, sino además cristalizó un sistema económico que consolidó a los Estados Unidos de América como el centro hegemónico del desarrollo y al resto de los países como instancias periféricas, aunque con la emergencia de las economías asiáticas, la geopolítica se redefinió a favor de Japón y Corea frente al declive de Europa después de la segunda guerra mundial.

Es en este contexto geopolítico y económico en el que se firmaron acuerdos y tratados para la reducción de emisiones de carbono, pero regulados por la dinámica de los países centrales con respecto a su influencia sobre los países periféricos [26]. En el marco de la pérdida de rectoría del Estado y la transformación de sus instituciones, el sistema de tarifas fue un instrumento de gestión que demostró la importancia de una administración consensuada de los recursos naturales y servicios públicos $\lceil 27\rceil$. De este modo, la política del cambio climático no sólo exacerbó las diferencias entre los actores políticos y civiles, sino además generó movilizaciones sociales y acciones colectivas a favor de la preservación del medio ambiente, la promoción de los derechos ecológicos y la defensa de los pueblos originarios.

Se trata de fenómenos colectivos que por su grado de organización enfocaron el problema en las políticas e instrumentos de gestión más que en la propaganda difundida en los medios a favor del desarrollo económico y el consumismo a partir de la explotación de los recursos naturales sin importar las capacidades de las generaciones futuras o la vulnerabilidad de las especies animales y vegetales $\lceil 28]$. El estudio de los fenómenos colectivos fue la primera aproximación teórica que trató de vincular las problemáticas ecológicas con la corrupción política y la propaganda de los medios de comunicación cristalizados en una agenda pública $\{29\rfloor$. El antropocentrismo y el clientelismo, aunada a la hipermetropía reflejaron el grado de crisis ambiental, económica, política y social en el que la humanidad se ubica frente al cambio climático.

Las teorías de los movimientos sociales y fenómenos de acción colectiva sugieren que el problema ya no estriba en el desbalance entre disponibilidad de recursos y necesidades de consumo, ni en la corrupción inherente al Estado y sus instituciones, sino en la difusión informativa que determina la indefensión, racionalidad o emotividad de la sociedad civil frente a la naturaleza y el Estado $\{30\rfloor$. En este tenor, las teorías y marcos conceptuales suponen que la acción colectiva es resultado de dos procesos, uno racional, deliberado, planificado y sistemático en el que la toma de decisiones depende de la disponibilidad de recursos, así como la movilización depende de las demandas logradas o cuando menos incluidas en la agenda civil[31]. Por consiguiente, cuando el proceso racional es inconsistente, emerge otro proceso más emotivo y afectivo en el que se incrementa la percepción de riesgo e inseguridad para dar cabida a la desesperanza, enojo, miedo, indignación o zozobra.

Se genera una identidad sociopolítica en la que la ciudadanía atribuye la responsabilidad a sus autoridades y representantes más que a sus posibilidades, oportunidades y capacidades de organización, acción debate y consenso [32]. La hipermetropía resultante exacerba la desesperanza, ya que una vez difundida la información relativa a inundaciones o sequías la sociedad civil genera creencias de escasez y desabastecimiento de agua o electricidad [33]. Acto seguido, las conductas de ahorro y cuidado emergen en los estilos de vida urbanos o comunitarios.

Sin embargo, desde la psicología se han propuestos teorías que tratan de explicar la acción de grupos organizados frente a las inundaciones o sequías difundidas o no en los medios, experimentadas o no en el entorno[34]. Las teorías psicológicas sostienen que la categorización de la información difundida en los medios es el primer eslabón determinante del comportamiento sustentable indicado por 
la prevención, conservación u optimización de los recursos $\{35\rfloor$. La teoría de la propensión al futuro advierte que una comunidad es sustentable siempre y cuando anticipe escenarios de escasez y desabastecimiento de agua o electricidad, pero no desde una estrategia de conservación, sino desde un estilo innovador en el que se buscan fuentes alternas de hidratación y energía.

Sin embargo, la teoría del comportamiento planificado advierte que ante los fenómenos globales las acciones locales son insuficientes, ya que la información diseminada en los medios es insuficiente para estructurar una acción efectiva que preserve los recursos y garantice un nivel de bienestar personal o grupal suficiente $\{36]$. En este mismo sentido, la teoría de la acción razonada se pronuncia a favor de delimitar las creencias informativas en decisiones de consumo, aunque no explicita la relación entre las actitudes hacia el desabastecimiento de agua o electricidad con respecto al desarrollo de una innovación [37]. Una propuesta más reciente advierte que los fenómenos naturales son considerados eventos impredecibles e inconmensurables que no podrían generar más que estrategias de optimización de los recursos.

La teoría de la percepción de riesgo plantea que las decisiones de consumo no dependen de la escasez o abundancia de recursos, sino de la relación entre disponibilidad y capacidades $\{38\rfloor$. En este sentido, una comunidad que percibe riesgos significativos de afectación a su salud por inundaciones o sequías pueden generar estrategias que le permitan subsistir aún y cuando su entorno los limite $[39]$. En un sentido similar, la teoría del procesamiento espontáneo sostiene que las experiencias de comunidades con inundaciones o sequías define una memoria colectiva que activa disposiciones a favor o en contra de medidas preventivas o colaborativas.

De este modo, la exposición histórica a los fenómenos naturales propicia una estructura de representaciones sociales que determina las percepciones de riesgo $u$ oportunidades como lo señala la teoría de la exposición social $[40]$. Ambos procesos, racional y emotivo son considerados por la teoría de la probabilidad de la elaboración para explicar la necesidad de información de comunidades que están al pendiente de la difusión de los medios y a partir de tales contenidos reaccionan o previenen afectaciones a grupos vulnerables $\{41]$. Las teorías y marcos conceptuales que tratan de explicar los efectos del cambio climático sobre la salud pública y el desarrollo endógeno coinciden en que el comportamiento favorable a la sustentabilidad es su indicador por excelencia, pero si más bien la sociedad civil genera indefensión y emotividad sociopolítica, entonces será posible observar conflictos que exacerben los efectos del cambio climático en la calidad de vida y bienestar personal.

\section{Estado del conocimiento}

Los estudios sociológicos y psicológicos relativos a los efectos del cambio climático sobre la salud pública resaltan las relaciones de dependencia entre variables explicativas del comportamiento humano, individual como grupal $\lceil 42\rfloor$. De este modo, las creencias entendidas como categorizaciones de información circundante del cambio climático están vinculadas con variables emocionales como el enojo o el miedo y con variables normativas [43]. La interrelación entre estas supone correlaciones con actitudes o percepciones $\{44\}$. Al ser las actitudes evaluaciones del entorno y las percepciones expectativas de la calidad del entorno, ambas están relacionadas con la toma de decisiones que supone las intenciones de llevar a cabo comportamientos de cuidado o ahorro en el consumo de los recursos naturales.

Sin embargo, los estudios advierten relaciones espurias entre las variables emocionales y las creencias, las actitudes y las percepciones $\{45\rfloor$. En el mismo sentido, las variables normativas tienen vínculos poco significativos con las intenciones y los comportamientos $\{46]$. Es decir, el enojo y el miedo que suponen las sequias e inundaciones no parecen estar relacionadas con las decisiones precautorias y los comportamientos de ahorro de los recursos.

En síntesis, los estudios psicológicos advierten que sólo variables de orden cognitivo pueden conforman un sistema deliberado, planificado y sistemático capaz de explicar al comportamiento humano como resultado del procesamiento de información relativa al cambio climático \47 \.

\section{Especificación de un modelo de variables observables}

La especificación de un modelo supone el establecimiento de las relaciones de dependencia entre sus factores $\{48\rfloor$. A partir de la revisión de la literatura, los marcos teóricos, conceptuales y empíricos, es posible delimitar un modelo.

Sin embargo, a diferencia de una red nomológica en la que se reflejan las relaciones establecidas por hipótesis debidamente contrastadas, en la especificación del modelo es posible innovar y por consiguiente, modificar las trayectorias de relaciones establecidas por la literatura $\{49\rfloor$. De este modo, el Desarrollo Local indicado por el comportamiento sustentable y las variables que explicitan el efecto de la información relativa al cambio climático sobre la toma de decisiones y la probabilidad de llevarlas a cabo, pueden ser ajustadas a las trayectorias establecidas.

En tal sentido, las percepciones o expectativas generadas por inundaciones y sequias pueden ser diversificadas en tres dimensiones según el grado de expectación a saber, utilidad de la información para la prevención de desastres, riesgos asociados al impacto de catástrofes en comunidades vulnerables y expectativas de control vinculadas a la reacción inmediata ante incendios o escasez de agua $\lceil 50\rfloor$. Cada una de las tres dimensiones perceptuales estarían asociadas a creencias y actitudes, o bien, a categorías y evaluaciones de la difusión de crisis ecológicas en los medios de comunicación. Ambas no sólo filtran la información, sino además la delimitan para que los individuos puedan tomar decisiones de evacuación, migración o permanencia. En cualquiera de los casos, la 
austeridad y ahorro de recursos estaría relacionada con la toma de decisiones.

\section{Discusión}

El presente trabajo ha especificado un modelo para el estudio del comportamiento favorable al Desarrollo ocal Sustentable. En relación con los marcos teóricos, conceptuales y empíricos, el presente modelo advierte que las Políticas del Cambio Climático, en sus rubros de desastres naturales y catástrofes ambientales, materializadas en inundaciones, sequías, incendios o desabastecimientos, podrían incorporar una estrategia comunicativa considerando la austeridad o el ahorro de agua.

No obstante, en virtud de que el modelo propuesto explicita el proceso deliberado, planificado y sistemático de la prevención de crisis relativas a la salud pública, es menester incorporar dos variables que el estado del conocimiento ha establecido, pero cuyas relaciones no son significativas con las variables incluidas en el modelo.

Se recomienda construir los instrumentos que permitan relacionar a las emociones y normas con percepciones, creencias, actitudes, intenciones y comportamientos. La validez y confiabilidad de los instrumentos permitirá elaborar un modelo integral a partir del cual la comunicación de riesgos y el desarrollo local no se entiendan como procesos opuestos, sino complementarios.

\section{Conclusión}

El aporte del presente trabajo al estado del conocimiento teórico, conceptual y empírico consiste en la especificación de un modelo, pero la inclusión de variables emocionales y normativas es indispensable para vincular la agenda de seguridad pública con la estrategia de Desarrollo Local.

\section{Referencias}

1. Abramo P. The city com -fusa: market and production of the urban structure in large Latin American metropolises. Eure, 2012: 38 (114) 35-69

2. Acosta A. Only by imagining other worlds, will this one be changed. Reflections on good living. Sustainability, 2010: 2, 5-21

3. Ajzen I, Joyce N, Sheikh S, Cote N. Knowledge and the prediction of behavior: the role of information accuracy in the Theory of Planned Behavior. Basic Application Social Psychology, 2011: 33, 101-117

4. Albacerrín D, Wallace H, Hart W. How judgments change following comparison of current and prior information. Basic Application Social Psychology, 2012: 34, 44-55

5. Albacerrín D, Wyer R. Elaborative and non-elaborative processing of a behavior related communication. Perspective Social Psychology Bulletin, 2011: 27, 691-705.

6. Barkin D, Lemus B. The ecological solidarity economy. A proposal in the face of our crisis. Sustainability, 2011: 5, 4-10

7. Beck A, Sinatra G, Lombardi D. Leveraging higher education instructor in the climate literacy effort: factors related to university faculty's propensity to teach climate change". International Journal Climate Change \& Research, 2013: 4 (4), 1-17.

8. Behancourth, L. Green consumers and the promotion of green markets: an alternative towards wellness, spirit, mind and health from the adoption of healthy lifestyles. Eleuthera. 2010: 4, 193-210
9. Berroeta H, Ramoneda A, Rodríguez V, Di Masso A, Vidal T. Apego al lugar, identidad del lugar, sentido de comunidad y participación cívica en personas desplazadas de la ciudad de Chaitén. Magic, 2015: $43 \quad$ (3), 51-63 https://scielo.conicyt.cl/pdf/magallania/v43n3/art05.pdf

10. Bertoni, M. and López, M. Values and attitudes towards the conservation of the biosphere reserve. Student Perspective. 2010: 19, 835-849

11. Blunda Y. Perception of volcanic risk and knowledge of emergency plans in the surroundings of the Poas volcano, Costa Rica. Geography Journal Central America. 2010 43, 201-209

12. Boza S. Los sistemas participativos de garantía en el fomento de los mercados locales de productos orgánicos. Polis, 2013: 12, (34), 1530

13. Carosio A. The culture of consumption against the sustainability of life. Sustainability, 2010: 2, 39-52

14. Carr W, Patterson M, Yung L, Spencer D. The faithful skeptics: evangelical religious belief and perception of climate change. JSRNC, 2012: 3, pp. 276-299.

15. Carreón J, Hernández J, García C, Bustos J, Morales M, Aguilar J. La psicología de la sustentabilidad hídrica: Políticas públicas y modelos de consumo. Apology, 2014: 63, 1-29 https://www.redalyc.org/pdf/4959/495950259004.pdf

16. Carreon J, Juarez M, García C. Governance of water sustainability: Theories and specification of co-management of local resources and services. Administrative Science, 2017: 1, 41-55

17. Carreon J, Sánchez A, Molina H, García M, Rojano S. Contrast of a quality control model for a sustainability in a Mexican organization in central Mexico. International Journal Environmental Agricultural Biollogy, 2017: 2 (6), 2908-2916

18. Corral V, Domínguez R. The role of antecedent and consequent events in sustainable behavior. Mexican Journal Behabior Analysis. 2011: 37, 9-29

19. Corral V, García F, Tapia C, Fraijo B. Sustainable behaviors and perceived psychological restoration. Acta Investoigación Psicológica, 2012: 2 (2), pp. 749-764.

20. Corral V, Mireles J, Tapia C, Fraijo B. Happiness as correlate of sustainable behaviors: a study of pro-ecological frugal, equitable and altruistic actions that promote subjective wellbeing". Research Human Ecology, 2011: 18 (2), pp. 95-104.

21. Corral V, Tapia C, Ortiz A, Fraijo B. Las virtudes de la humanidad, justicia y moderación y su relación con la conducta sustentable. Revista Internacional Psicología, 2013: 45 (3), pp. 361-372.

22. Cortés H, Peña I. De la sostenibilidad a la sustentabilidad, modelo de desarrollo sustentable para su implementación en políticas y proyectos. Revista Admistración \& Negocios, 2015: 78, 40-54 https://www.redalyc.org/pdf/206/20640430004.pdf

23. Coulomb R. Sustentabilidad de la centralidad urbana e histórica. Una reflexión desde el centro histórico de la Ciudad de México. Quic, 2008: $\quad 10 \quad$ (2), 29-49 https://www.redalyc.org/pdf/401/40113196003.pdf

24. Cunsolo A, Harper S, Ford J, Edge V, Ladman K, Houle K, Blake S, Wolfrey C. Climate change and mental health: an exploratory case study from Rigolet, Nunatsiavut, Canada. Climate Change, 2013: 120, (12), 255-270.

25. Dasaklis T, Pappis C. Supply chain management in view of climate change: an overview of possible impacts and the road ahead. Journal Independence, 2013: 6 (4), 1124-1138.

26. Duerden $\mathrm{M}$, Witt $\mathrm{P}$. The impact of direct and indirect experiences on the development of environmental knowledge, attitudes and behavior. Journal Environmental Psychology, 2010: 30, 379-392

27. Flores M, Parra M. Characterization of domestic water savings in the Murcia region based on sociodemographic components. Consensus Social Science. 2011: 13, 1-13

28. Fraijo B, Corral V, Tapia C, García F. Adaptación y prueba de una escala de orientación hacia la sustentabilidad en niños de sexto año de educación básica. Revista Mexicana Investigación Educativa, 2012: 17 (55), 1091-1117.

29. Frías M, Corral V. Environmental and individual factors in adolescent anti-sociality: a structural model of Mexican teenagers. International Journal Critical Justice Science, 2013: 8 (2), 198-214. 
30. Galicia L, Balderrama J, Navarro R. Validez de contenido por juicio de expertos: propuesta de una herramienta virtual. Ape, 2017. 9 (2), 42-53 http://dx.doi.org/10.32870/Ap.v9n2.993

31. García C, Juárez M, Bustos J. The journalistic coverage around the conflicts due to the shortage of water in Mexico, Federal District. Artic, 2017: 41, 93-128

32. García C, Juárez M, Sandoval F, Bustos J. A psychological approach to environmental complexity: Specification of a community stress and resilience model. Communication, 2017: 14, 75-95

33. García C, Rivera B, Limón G. Perception model sustainability of the generation millennials. Inclusion, 2017: 4 (4), 82-96

34. García C. La exclusión hidrológica. Revista Internacional Entelequia, 2010: 11, 41-59.

35. García C. Modelo especificado para el estudio de los efectos del cambio climático sobre la salud pública. Revista Científica

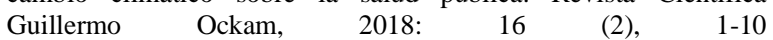
https://www.redalyc.org/jatsRepo/1053/105358033006/html/index. html

36. García R, Márquez H. México: Violencia e inseguridad. Hacia una estrategia de desarrollo y seguridad humana. Psychology Ecological, 2013: Número especial, $1-30$ https://www.redalyc.org/pdf/181/18127008015.pdf

37. Gaxiola J, Frías M, Figuerero J. Factores protectores y los estilos de crianza: un modelo bioecológico. Revista Mexicana de Investigación en Psicología Social y de la Salud, 2020: 1, pp. 28-40.

38. Henríquez C, Guzmán J, Salcedo D. Minería de opiniones basado en la adaptación al español de ANEW sobre opiniones acerca de hoteles. Prolegomenum, 2016:56, 25-32 https://www.redalyc.org/pdf/5157/515754423002.pdf

39. Martínez A, Porcellli A. Un difícil camino en pos del consumo sustentable: el dilema entre la obsolescencia programada, la tecnología y el ambiente. Lex, 2016: 18 (14), 333-378

40. Martínez R, Martínez D. Perspectivas de la sustentabilidad: teoría y campos de análisis. Acta, 2016: 16 (26), 123-145

41. Medrano L, Muñoz R. Aproximación conceptual y práctica a los modelos de ecuaciones estructurales. Revista Digital Investigación Documental Universitaria, 2017: 11 (1), 219-239 http://dx.doi.org/10.19083/ridu.11.486

42. Nava L, Gandín J. Geopolítica del medio ambiente: cambio climático y recursos hídricos, aproximaciones al caso de Canadá. Aba, 2015: 3 (85), 121-135

43. Nieto F. El legado del sexenio de la corrupción: los retos del sistema nacional anticorrupción. Frontiers International, 2020: 40 (2), 683715 http://www.scielo.org.mx/pdf/fi/v60n2/0185-013X-fi-60-02683.pdf

44. Nozica G. Plan for territorial integration. The desirable scenarios for the insertion of the province of San Juan into Mercosur. IberoAmerican Journal Urbanism. 2011: 6, 43-54

45. Quiroz D. Cities and climate change: the case of Mexico City's climate policy. Dem Urb Stu, 2013: 28 (83), 343-382

46. Restrepo J. El concepto de riesgo: Avances hacia un modelo de percepción de riesgos en la salud. Psicologia, 2016: 10 (16), 174-200 https://dialnet.unirioja.es/servlet/articulo?codigo $=5578187$

47. Sandoval F, Carreon J, García C, Quintero M. Bustos J. Model of the determinants of the perception of resilience based on perceived risk and stress in relation to the governance of civil protection. Invurnus, 2017: 12 (1), 30-35

48. Sandoval I. Enfoque de la corrupción estructural: poder, impunidad y voz ciudadana. Revista Mexicana Sociología, 2016: 78 (1), 119152 http://www.scielo.org.mx/pdf/rms/v78n1/0188-2503-rms-7801-00119.pdf

49. Sharples D. Communicating climate science: evaluating the UK public's attitude to climate change. Earth Environment, 2010: 5, 185205

50. Uribe I. D, Gallo L, Fernández A. Trazos de una educación hedonista. Movilización, 2017: 23 (1), 339-349 https://www.redalyc.org/pdf/1153/115350608024.pdf 